\title{
The importance of non-photon noise in stellar spectropolarimetry
}

\section{The spurious detection of a non-existing magnetic field in the $\mathrm{A} 0$ supergiant HD 92207}

\author{
S. Bagnulo ${ }^{1}$, L. Fossati ${ }^{2}$, O. Kochukhov ${ }^{3}$, and J. D. Landstreet ${ }^{1,4}$ \\ 1 Armagh Observatory, College Hill, Armagh BT61 9DG, Northern Ireland, UK \\ e-mail: sba@arm.ac.uk \\ 2 Argelander-Institut für Astronomie der Universität Bonn, Auf dem Huegel 71, 53121 Bonn, Germany \\ e-mail: lfossati@astro.uni-bonn.de \\ 3 Department of Physics and Astronomy, Uppsala University, 75120 Uppsala, Sweden \\ e-mail: oleg.kochukhov@physics.uu.se \\ ${ }^{4}$ Physics \& Astronomy Department, The University of Western Ontario, London, Ontario N6A 3K7, Canada \\ e-mail: jlandstr@uwo.ca
}

Received 18 July 2013 / Accepted 26 August 2013

\begin{abstract}
Context. The low-resolution, Cassegrain mounted, FORS spectropolarimeter of the ESO Very Large Telescope is being extensively used for magnetic field surveys. Some of the new discoveries suggest that relatively strong magnetic fields may play an important role in numerous physical phenomena observed in the atmospheres as well as in the circumstellar environments of certain kinds of stars. Aims. We show in detail how small instabilities or data-reduction inaccuracies represent an alternative explanation for the origin of certain signals of circular polarisation published in recent years.

Methods. With the help of analytical calculations we simulate the observation of a spectral line in spectropolarimetric mode, adding very small spurious wavelength shifts, which may mimic the effects of seeing variations, rapid variations of the stellar radial velocity, or instrument instabilities. As a case study, we then re-visit the FORS2 measurements that have been used to claim the discovery of a magnetic field in the A0 supergiant HD 92207. In addition, we present new observations of this star obtained with the HARPSpol instrument.

Results. Both calibration and science data show compelling evidence that photon-noise is not the only source of error in magnetic field measurements, especially in sharp spectral lines. Non-photon noise may be kept under control by accurate data reduction and quality controls. Our re-analysis of FORS2 observations of HD 92207 shows no evidence of a magnetic field, and we are able to reproduce the previous FORS detection only by degrading the quality of our wavelength calibration. Our HARPSpol spectropolarimetric measurements show no evidence of a magnetic field at the level of $10 \mathrm{G}$.

Conclusions. Our work contributes to a better understanding of the importance of accurate data treatment and instrument characterisation, and demonstrates that ultra-high signal-to-noise ratio measurements do not automatically translate into ultra-high accuracy.
\end{abstract}

Key words. stars: magnetic field - polarization - techniques: polarimetric - stars: individual: HD 92207

\section{Introduction}

Polarimetry is attracting a steadily growing level of interest in various research areas of astronomy. The most popular and successful application of spectropolarimetery is to magnetic stars, but polarimetric techniques may make significant contributions to the advance of knowledge in many other fields. Perhaps not surprisingly, the most interesting applications are those that require measurements at the very limit of the present and near future instrumentation, for example the characterisation of the atmospheres of exo-solar planets, and the search for extraterrestrial life (Sterzik et al. 2012).

Even in the traditional area of stellar magnetism, much of the most interesting work recently has been directed to the detection of very weak fields in a variety of stars that in the past were thought to be non-magnetic, or in which fields were undetectable. Fields have recently been discovered in a variety of types of stars such as Of?p stars (Grunhut \& Wade 2012, and references therein), $\beta$ Cep and slowly pulsating B stars (Henrichs et al. 2000; Neiner et al. 2003; Silvester et al. 2009), Herbig AeBe stars (Wade et al. 2005, 2007; Catala et al. 2007; Alecian et al. 2013), red giant (Aurière et al. 2009) and AGB supergiant stars (Grunhut et al. 2010), and many kinds of cool dwarfs (Donati \& Landstreet 2009, and references therein). Fields in such stars have become detectable as a result of having new generation instrumentation of very high efficiency and broad wavelength coverage available on medium and large telescopes, and through advanced data reduction and treatment techniques that allow very small polarimetric signatures to be reliably detected. However, at the same time that these new techniques have made possible the discovery of magnetic fields in many stars, the difficulties of reducing the data and evaluating the uncertainties of 

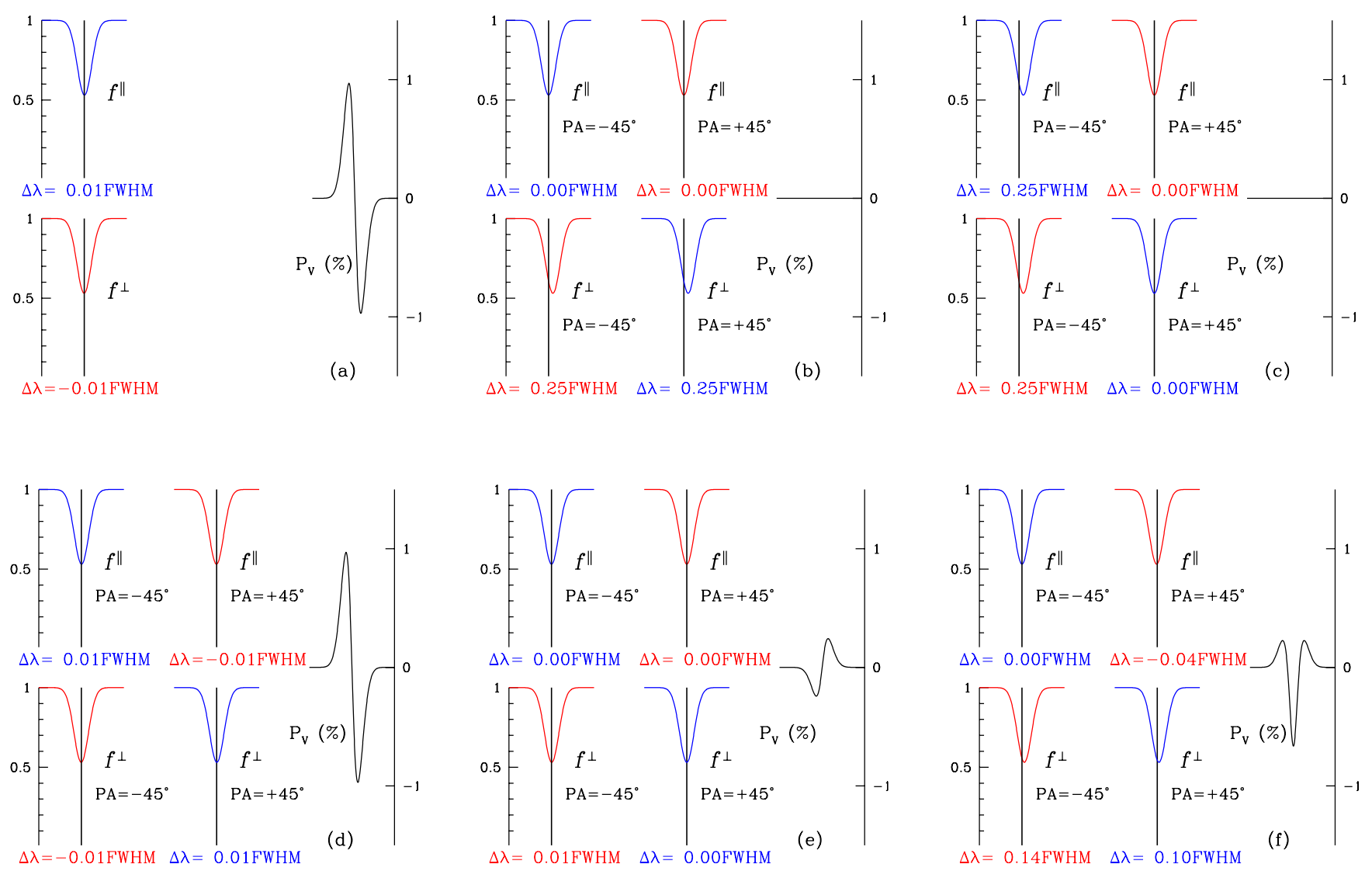

Fig. 1. Combinations of Gaussian profiles to simulate polarimetric observations of a spectral line. The figure is explained in the text.

measurement correctly have led to a number of reports of discoveries of fields in stars in which fields have not in fact been detected (Bagnulo et al. 2012).

The aim of this paper is to discuss how easily spurious signals can appear in polarimetric observations, and to discuss some general considerations that may be relevant when designing new instrumentation and reduction pipelines for high-precision spectropolarimetry. We will try to clarify some specific sources of error and uncertainty in high-precision spectropolarimetry that we believe have contributed significantly to previous incorrect reports of field detections. This work may therefore be applicable to a wider domain than simply that of stellar magnetism, because of the expanding relevance of spectropolarimetry (including linear spectropolarimetery) to many domains. We hope that this discussion may contribute to significantly reducing the number of dubious or erroneous reports of field discoveries appearing in the literature.

In this paper we present in Sect. 2 a set of simple numerical simulations that illustrate some important issues about instrument stability and wavelength calibration. In Sect. 3 we analyse some calibration and science data obtained with the FORS lowresolution spectropolarimeter of the ESO VLT, which is one of the most active instruments in the search for stellar magnetism. These data allow us to estimate the size of some kinds of data uncertainty in observations. In Sect. 4 we re-discuss FORS observations of the A0 supergiant star HD 92207 that have led to the claim by Hubrig et al. (2012) of the discovery of a new class of magnetic stars, and we present new observations of HD 92207 obtained with the HARPSpol spectropolarimeter at the $3.6 \mathrm{~m}$ telescope of the ESO La Silla Observatory. In Sect. 5 we discuss our results and present our conclusions.

\section{The effects of the beam-swapping technique on wavelength shifts}

There are different physical mechanisms that may be responsible for the circular polarisation of a spectral line. The Zeeman effect causes a differential offset of a spectral line observed in opposite polarisations.

To simulate the effects of small instrument flexures during the observation of a spectral line in polarimetric mode, we may combine Gaussian profiles after they are offset by a small fraction of their FWHM. Figure 1 shows various examples of such combinations, considering Gaussians with $F W H M=1$ and depth $=0.5$. Case (a) is the simple combination of two Gaussian profiles offset by $\pm 0.01 F W H M$; the profile on the right-hand side of panel (a) is given by the ratio between the difference and the sum of the two profiles on the left-hand side, $\left(f^{\|}-f^{\perp}\right) /\left(f^{\|}+f^{\perp}\right)$, expressed in per cent units. This case may represent a polarised spectral line observed in the parallel $\left(f^{\|}\right.$, upper line) and in the perpendicular $\left(f^{\perp}\right.$, lower line) beam after the light has travelled across a retarder waveplate and a Wollaston prism. In case of circular polarisation, the parallel beam has the right-hand circularly (RHC) polarised profile, the perpendicular beam has the left-hand circularly (LHC) polarised profile (in all panels of Fig. 1, the RHC flux is represented by blue lines, and the LHC flux is represented by red lines). This case could also represent the observation of a nonpolarised spectral line where the offsets have been artificially introduced by e.g., a slightly incorrect wavelength calibration of the parallel and perpendicular beams.

In the remaining cases we simulate the beam swapping technique, which is commonly employed in real polarimetric 
observations. If the $\lambda / 4$ retarder waveplate is rotated by $90^{\circ}$, then the signal in the parallel beam becomes proportional to the LHC polarised light, and the signal in the perpendicular beam is proportional to the RHC polarised light. The reduced $P_{V}=V / I$ profiles are obtained with the formula

$P_{V}=\frac{1}{2}\left[\left(\frac{f^{\|}-f^{\perp}}{f^{\|}+f^{\perp}}\right)_{-45^{\circ}}-\left(\frac{f^{\|}-f^{\perp}}{f^{\|}+f^{\perp}}\right)_{+45^{\circ}}\right]$.

The advantages of this technique are that many spurious effects cancel out, as originally explained in the FORS user manual, and explained in greater detail by Bagnulo et al. (2009). Case (b) of Fig. 1 shows that if the wavelength shift between parallel and perpendicular beams is an instrumental or a calibration artifact which remains constant as the retarder waveplate rotates, then its effect is canceled out if the fluxes are combined as prescribed by Eq. (1), and the resulting reduced Stokes profile is flat. Similarly, the effect of an offset that is present in both parallel and perpendicular beams only at one position of the retarder waveplate (case c) is canceled out. If the wavelength shift is due to the polarisation intrinsic to the spectral line, then the offset between top and bottom beam is reversed when the retarder waveplate is rotated by $90^{\circ}$ (case d), and the combination given by Eq. (1) reproduces a genuine Zeeman feature. Finally, cases (e) and (f) show that an artificial offset between spectral lines in opposite polarisations, which appears or changes after the retarder waveplate rotates, may be responsible for a spurious polarisation signal.

\section{The occurrence of spurious offsets and their impact on science data}

To discuss the likelihood of the occurrence of spurious offsets in real observations, we need to investigate the spectral image stability and compare it to the FWHM of the spectral lines used for magnetic field detection.

\subsection{Offsets introduced by the polarimetric optics}

To have an approximate idea of the offsets potentially involved in practical applications, we first consider calibration data obtained with the FORS instrument in spectropolarimetric mode, and we estimate the size of the offsets introduced by the polarimetric optics. In particular, we inspect the images of the arc lines used for wavelength calibration with the grating $600 \mathrm{~B}$, when the CCD is read out with no rebinning. Pixel size is $15 \mu \mathrm{m}$, which corresponds to a pixel scale of $0.125^{\prime \prime}$ on sky. Instrument dispersion is $\sim 0.75 \AA$ per pixel. All data considered in the paper were obtained with the E2V CCD mosaic, but the geometrical characteristics are identical for the more commonly used MIT CCD mosaic.

We recall that calibrations are taken during day time with the telescope at zenith, using the inner side of the Cassegrain shutter as a screen. This implies that slit illumination is constant from one calibration arc to the next, which is not the case for stellar observations.

We look at the 400 lower rows of the upper chip, which is the region used for most of the science observations when only one target is observed. Specifically, the perpendicular beam is recorded in the region around pixel lines $\sim 100-150$ pixels, while the parallel beam is recorded in the region around pixel lines $\sim 290-340$. Figure 2 shows a detail of the tracing of the arc $\mathrm{Hg}$ I line at $\lambda=4358.343 \AA$, obtained in different exposures with

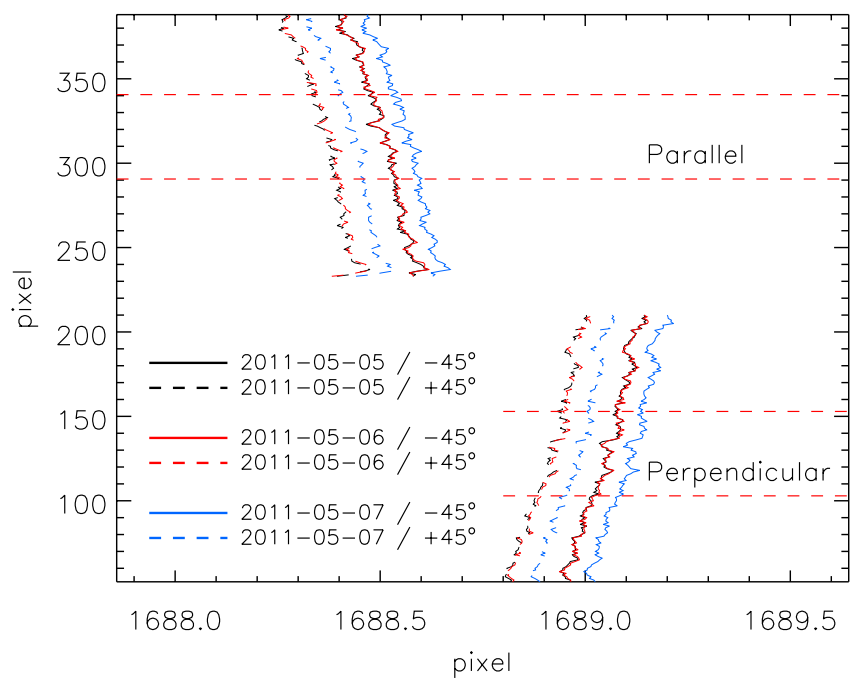

Fig. 2. Tracing of the centre of the Hg I arc line at $\lambda=4358.343 \AA$ on the FORS2 detector in the spatial region where science spectra are recorded. Different colours and line-styles refer to calibration frames obtained on different days and with different positions of the retarder waveplate, as explained in the text. Note that black and red lines are practically superposed, but the line positions on 2011-05-07 are offset by $\sim 0.08$ px with respect to those measured on 2011-05-05 and 2011-05-06.

the retarder waveplate at position angles $-45^{\circ}$ and $+45^{\circ}$, and on three consecutive days. The tracing was carried out by fitting the line profile in each pixel row (the $x$-axis of the CCD) with a Gaussian profile, and then by following the Gaussian peaks along the direction perpendicular to the dispersion (the $y$-axis of the CCD). The arc line FWHM is 3.3 pixels, for a spectral resolution of $\Delta \lambda=2.5 \AA$.

It appears that, due to field distortion, and in particular due to the fact that the beams split by the Wollaston follow different optical paths, the perpendicular (bottom) beam is redshifted by approximately 0.55 px (i.e., about $15 \%$ of the line FWHM) with respect to the parallel (top) beam. When the retarder waveplate is rotated from $-45^{\circ}$ to $+45^{\circ}$ all beams are blue-shifted by $\sim 0.13$ px ( $\sim 4 \%$ of the line FWHM). This very small offset is likely due to the fact that the retarder waveplate does not rotate in a plane perfectly perpendicular to the incoming light beam. Line positions are approximately, but not perfectly, reproduced when the retarder waveplate is rotated, removed and re-inserted at different epochs - this is likely due to a combination of flexures/hysteresis of the system telescope+instrument. Such offsets, if not corrected, would generate a spurious signal of polarisation qualitatively similar to that of Fig. 1 (case f).

The risk of spurious artefacts may be minimised with a very accurate wavelength calibration, and certainly, accurate wavelength calibration may compensate for the offset between parallel and perpendicular beam. To take the shifts introduced when the retarder waveplate rotates into account, one could calibrate the science observations obtained with the retarder waveplate at a certain position angle using the arc frame obtained with the retarder waveplate at the same position angle, making sure that arc frames at $-45^{\circ}$ and $+45^{\circ}$ are obtained without having the retarder waveplate removed and re-inserted in between. This means that each observing set needs to be treated with at least four different wavelength calibration solutions. However, experience shows that the additional noise introduced by adopting two independent calibration frames may be responsible for larger artificial offsets than those that they are meant to be corrected 
(Bagnulo et al. 2006, 2009), potentially leading to spurious detections, such as those discussed by Jordan et al. (2012) and Landstreet et al. (2012). Of course, two independent solutions must be obtained for the two beams split by the Wollaston prism, and to keep the highest level of consistency, these two solutions must be obtained adopting exactly the same fitting function and using exactly the same arc lines. Once the offset between parallel and perpendicular beam is corrected with two independent wavelength calibrations, then we are in case (c) of Fig. 1, and the effect of the offset introduced when the retarder waveplate rotates should be fully compensated. Alternatively, one could simply apply a rigid offset of 0.13 px to all frames obtained with the retarder waveplate at $+45^{\circ}$ (which corresponds to a wavelength step that depends on the specific instrument setting). However, since day-time calibrations do not reproduce the exact optical path of the night-time science observations, we cannot confirm that is the best data reduction strategy.

\subsection{Offsets appearing in science spectra}

As a real science application, in this section, and in Sects. 4.1 and 4.2, we examine in detail the magnetic observations of the A0 supergiant HD 92207, for which Hubrig et al. (2012) have claimed discovery of a magnetic field on the basis of two apparently significant field detections, one of $-402 \pm 52 \mathrm{G}$ and one of $+157 \pm 51 \mathrm{G}$, using Balmer lines (slightly different results are obtained from the analysis of the full spectrum). We focus on the first of these two measurements, which is nominally significant at the $8 \sigma$ level. These observations were obtained on 2011-05-07 with the FORS2 instrument of the ESO VLT. The grism $600 \mathrm{~B}$ was used with a $0.4^{\prime \prime}$ slit width. FORS2 data reduction was performed independently by two of us (SB and LF) using various IRAF tasks and independent FORTRAN and IDL routines, and repeated using the ESO pipeline (Izzo et al. 2010).

Various simple tests can be devised to check the stability of the spectrum image. For instance, in the ideal case, a given line profile (normalised to the continuum) of a non-variable star, obtained under identical instrument settings, should appear constant within photon-noise error bars. The visual impact of a wavelength shift is clearly higher on deep and sharp lines than on shallow lines. Here we consider the Ca II K line at $\lambda=3933.7 \AA$, which, at the instrument resolution, appears as a deep and sharp feature, with a FWHM of about 3.6 pixels.

The four top panels of Fig. 3 show the profiles extracted from the parallel and perpendicular beams obtained at two positions of the retarder waveplate, prior to wavelength calibration. Different colours and line-styles are used for the profiles extracted during different exposures obtained in temporal sequence as given in the caption. Ideally, all profiles within each panel should coincide within photon-noise error bars, which is about the same size as the adopted symbols. However, even profiles obtained consecutively without moving the retarder waveplate, e.g. profiles 2 and 3, appear clearly shifted. The largest offset is the one observed between profiles 5 and 8 , both obtained with the retarder waveplate at $-45^{\circ}$ (two upper panels). The centre of the Gaussian used to approximate profile no. 8 is blue-shifted by 0.25 px with respect to the Gaussian that best fits the profile no. 5 .

The bottom panel of Fig. 3 shows the corresponding Stokes $I$ profiles. The profiles extracted in the parallel and in the perpendicular beam have been added together, after correcting for the shifts observed in Fig. 2, namely: all perpendicular beams have been offset by -0.55 pixels and all beams obtained with the retarder waveplate at $+45^{\circ}$ have been offset by $+0.13 \mathrm{px}$.
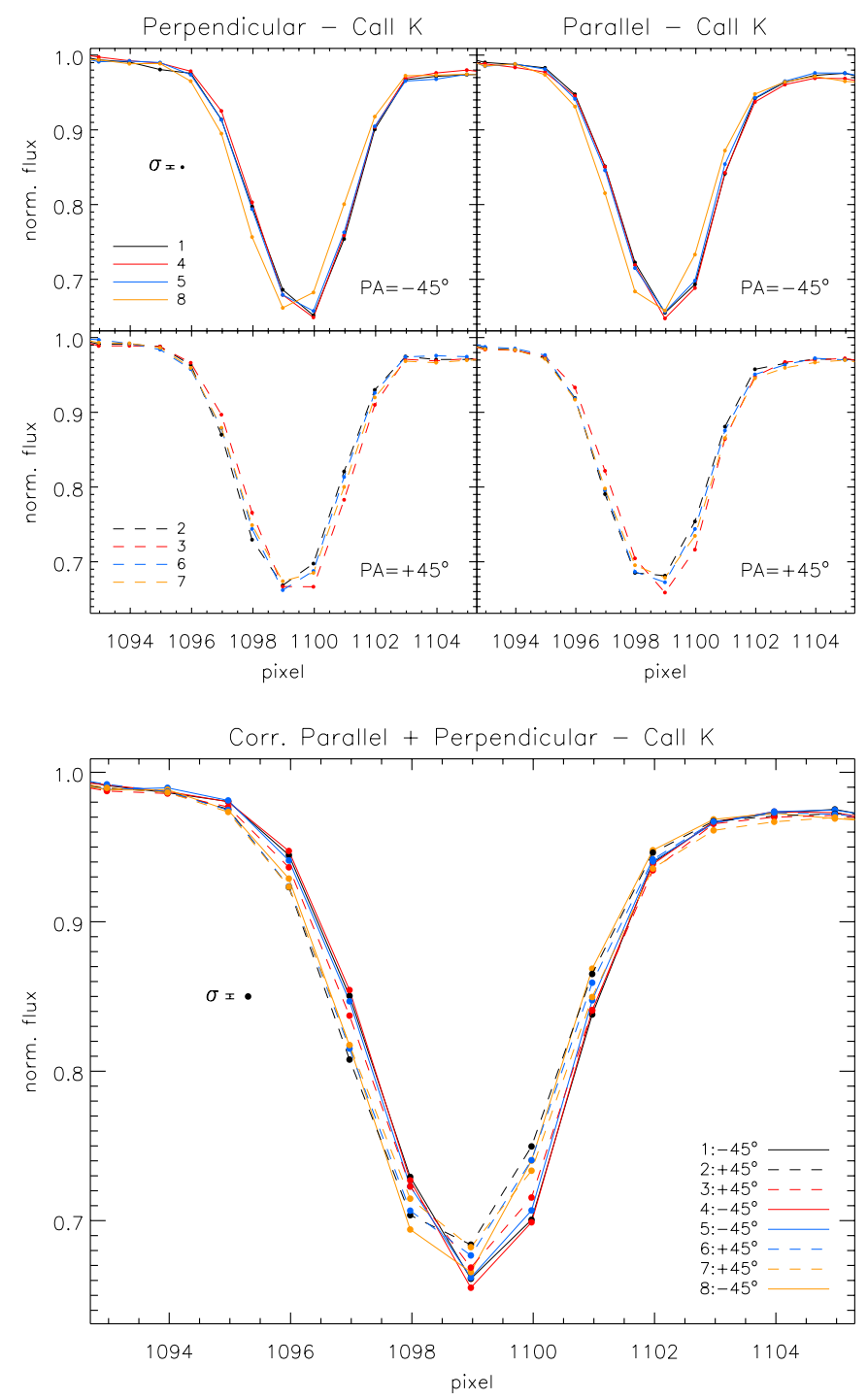

Fig. 3. Profiles of the Ca II K line extracted from the FORS frames of the night on 2011-05-07 as follows: 1: UT 03:56:53 $\left(-45^{\circ}\right)$; 2: UT 03:58:28 $\left(+45^{\circ}\right)$; 3: UT 03:59:32 $\left(+45^{\circ}\right)$; 4: UT 04:01:08 $\left(-45^{\circ}\right)$; 5: UT 04:02:12 $\left(-45^{\circ}\right)$; 6: UT 04:03:49 $\left(+45^{\circ}\right)$; 7: UT 04:04:54 $\left(+45^{\circ}\right)$; 8: UT 04:06:29 $\left(-45^{\circ}\right)$. Note that pairs $(2,3),(4,5)$ and $(6,7)$ were obtained consecutively without changing the instrumental setup. The retarder waveplate is rotated after the end of the exposures No. 1, 3, 5, and 7. The figure is explained in the text.

In the ideal case, regardless the presence of a magnetic field, all these profiles should be indistinguishable within photon-noise error bars. In contrast, the obvious presence of shifts shows that either the instrument or the star image on the slit are stable only within a fraction of a pixel (say $25 \%$, corresponding to $\sim 0.03^{\prime \prime}$ on sky). Our conclusions are that spectral lines in consecutive science exposures have an offset pattern more complex than that observed in the arc lines as the retarder waveplate rotates, and that the actual error bars on spectral lines are larger than those due to Poisson noise. This is consistent with and further supports a general finding by Bagnulo et al. (2012).

It is of some interest to display the combinations of the profiles of Fig. 3 according to Eq. (1). The leftmost panel of Fig. 4 shows the Ca II K profiles combined without wavelength calibration; the result is a deep spike with a $\sim 2 \%$ amplitude, qualitatively similar to panel (f) of Fig. 1. The middle panel shows the same combination after applying the offest correction measured 
S. Bagnulo et al.: The importance of non-photon noise in stellar spectropolarimetry

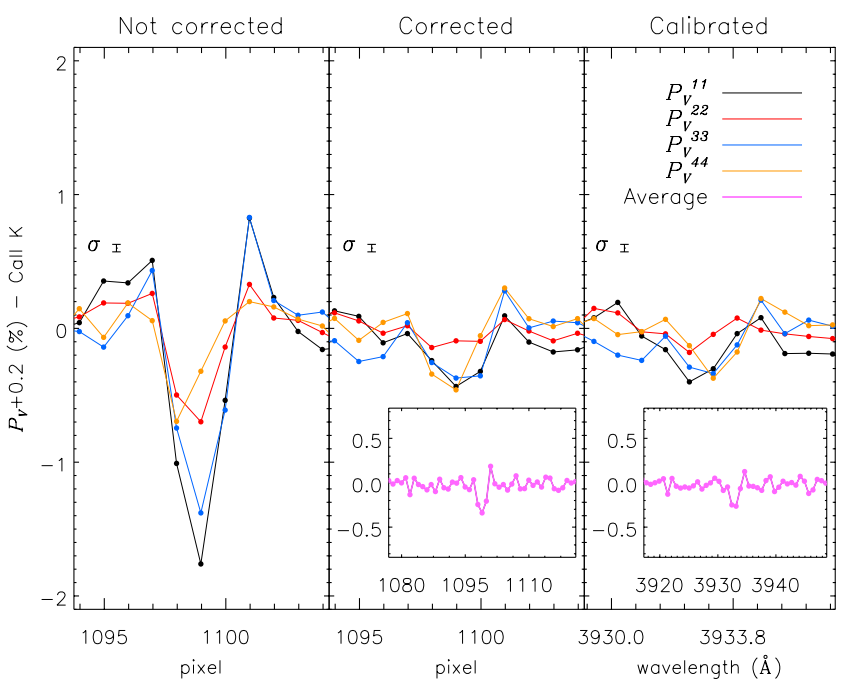

Fig. 4. $P_{V}$ profiles of the Ca II K line of the night 2011-05-07 combined as explained in the text. $P_{V}^{11}$ : UT 03:56:53 $\left(-45^{\circ}\right)+$ UT 03:58:28 $\left(+45^{\circ}\right) ; P_{V}^{22}$ : UT 04:01:08 $\left(-45^{\circ}\right)+\mathrm{UT}$ 03:59:32 $\left(+45^{\circ}\right) ; P_{V}^{33}: \mathrm{UT}$ 04:02:12 $\left(-45^{\circ}\right)+$ UT 04:03:49 $\left(+45^{\circ}\right) ; P_{V}^{44}$ : UT 04:06:29 $\left(-45^{\circ}\right)+\mathrm{UT}$ 04:04:54 $\left(+45^{\circ}\right)$. The insets provide an enlarged view of the average of the $P_{V}^{j j}$ profiles of the Ca II line.

on the arc line of Fig. 2. The feature in the "pseudo- $P_{V}$ " profiles is now greatly reduced. Finally, the rightmost panel shows the "true" $P_{V}$ profiles, i.e., obtained after a global wavelength calibration of the various profiles. A feature is still visible, but from our previous analysis we conclude that it is unlikely that it is due to the Zeeman effect. We finally note that a similar analysis carried out for other spectral lines lead to similar conclusions as that carried out for the Ca II line.

\section{The non-detection of a magnetic field in the AO supergiant HD 92207}

\subsection{FORS archive data}

The results of our reduction of FORS2 data (see Fig. 5) are significantly different from those published by Hubrig et al. (2012). First of all, our circular polarised profiles show a global offset of $\sim-0.2 \%$. This could be a symptom of cross-talk from linear to circular polarisation (Bagnulo et al. 2009): a fraction of the linear polarisation intrinsic to the source may be transformed into circular polarisation by the telescope or instrument optics, before reaching the polarimetric optics. Based on FORS1 linear polarisation observations, Ignace et al. (2009) reported for HD 92207 an average continuum polarisation of $P_{Q} \sim-2 \%$ and $P_{U} \sim-2.6 \%$. Therefore the continuum of circular polarisation could be explained as a $10 \%$ cross-talk from $P_{Q}$ to $P_{V}$, which, although a bit higher than expected, is not unrealistic. Another possible explanation (supported by simple numerical simulations) is the presence of scattered light that slightly changes from one exposure to the next.

Compared to the results by Hubrig et al. (2012), the most noticeable difference is however that, once the profile is rectified, our field measurement is consistent with zero: $\left\langle B_{z}\right\rangle=$ $-190 \pm 65 \mathrm{G}$; the rectification procedure, and the way the longitudinal field is calculated, are described in detail by Bagnulo et al. (2012), and the error bar is given using their Eq. (11), i.e., we have considered the external error; however, in this paper, we have not performed any clipping on the data. Following some
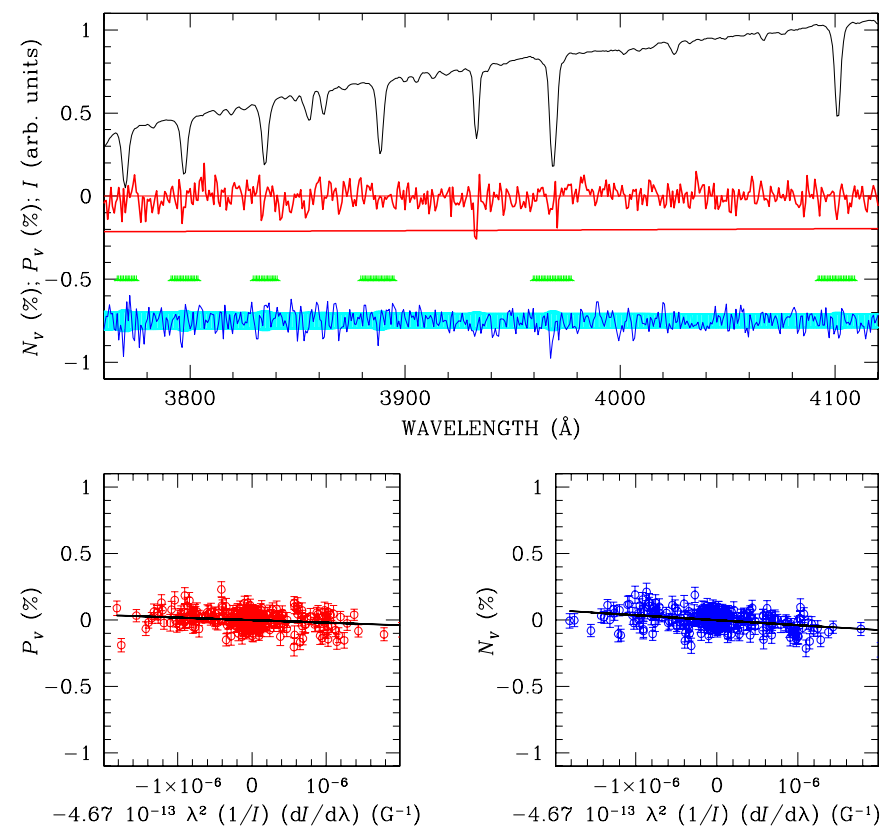

Fig. 5. Polarised spectrum of HD 92207 observed on 2011-05-07 with grism 600B. The top panel shows the observed Stokes I profile (black solid line, in arbitrary units, and not corrected for the instrument response), the rectified $P_{V}$ profile (red line centred about 0 ; the red smooth solid line slightly offset from zero shows the position of the $P_{V}$ "continuum" prior rectification) and the null profile (blue solid line, offset by $-0.75 \%$ for display purpose). The $P_{V}$ error bars are represented with a light blue band centred about $0.75 \%$. The slope of the interpolating lines in the bottom panels gives the mean longitudinal field from $P_{V}$ (left bottom panel) and from the null profile (right bottom panel), both calculated using the $\mathrm{H}$ Balmer lines only. The regions adopted to derive the magnetic field are shown by thick green lines centred around -0.5 . The corresponding $\left\langle B_{z}\right\rangle$ and $\left\langle N_{z}\right\rangle$ values are $-190 \pm 65 \mathrm{G}$ and $-375 \pm 65 \mathrm{G}$, respectively.

tendency in the literature, one might be tempted to describe our result as a $2.9 \sigma$ probable detection. However, our analysis of the profile instability presented in the previous section strongly suggests that this is instead simply a null detection, and that also the feature associated to the Ca II (visible in our plot, although less pronounced than that shown by Hubrig et al. 2012), is almost certainly spurious. A further indication that our " $2.9 \sigma$ detection" should not be treated as a marginal detection comes from the $5.7 \sigma$ field detection in the null profile: $\left\langle N_{z}\right\rangle=-375 \pm 65 \mathrm{G}$ (we recall that null profiles are expected to be oscillating about zero within error bar, and that, if used to measure the magnetic field, one should obtain a value consistent with zero).

It remains to understand why Hubrig et al. (2012), from the same dataset, obtained an $8 \sigma$ detection. This measurement, if correct, would represent a firm field detection (from very general statistical considerations, Bagnulo et al. (2012) concluded that FORS magnetic field detections are secure only if they reach at least a 5-6 $\sigma$ level). Aside from the fact that our data-reduction does not lead to a field detection, we point out that the shape of the $P_{V}$ profiles shown in Fig. 1 of Hubrig et al. (2012) can hardly be explained in terms of Zeeman effect. Unless the region of line formation is characterised by strong radial convective modes and the magnetic field changes with optical depth, the $P_{V}$ profiles formed in a magnetic atmosphere always have the zero-order moment equal to zero (i.e., the integral of Stokes $V$ over the region of one spectral line is equal to zero). In contrast, the $P_{V}$ profiles shown in Fig. 1 of Hubrig et al. (2012) seem to have a 

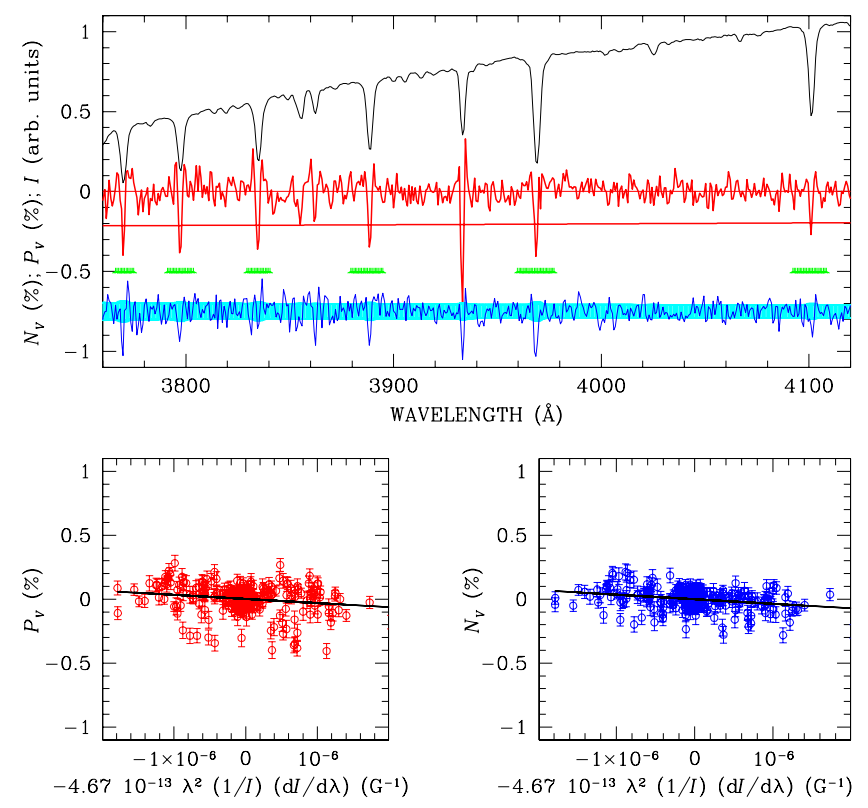

Fig. 6. As Fig. 5, but adopting a less than optimal wavelength calibration as explained in the text. Note that spikes are detected both in $P_{V}$ and in the $N_{V}$ profiles. The corresponding $\left\langle B_{z}\right\rangle$ and $\left\langle N_{z}\right\rangle$ values are $-325 \pm$ $105 \mathrm{G}$ and $-355 \pm 75 \mathrm{G}$, respectively.

strongly negative zero-order moment, and qualitatively resemble the $P_{V}$ profiles obtained by combining the non-wavelengthcalibrated beams shown in the left panel of our Fig. 4. Therefore we suggest that the field detection of Hubrig et al. (2012) is the result of the combined effect of a less than optimal wavelength calibration and a not perfectly stable spectral image on the CCD.

To support our hypothesis, in Fig. 6 we show the results of a different reduction of the same data, in which we have introduced a small difference in the way we have wavelength calibrated the parallel and the perpendicular beam. For the wavelength calibration of the parallel beam we have interpolated with a third order cubic spline 22 lines of the arc spectrum listed in the line catalogue of the FORS user manual. For the wavelength calibration of the perpendicular beam we have used a first order cubic spline, and we have not included two blue He I arc lines, one at $\lambda=3888.6 \AA$ and one at $\lambda=4026.1 \AA$. The $P_{V}$ profile that we have obtained using this less than optimal procedure is very similar to that presented by Hubrig et al. (2012) in the bottom panel of their Fig. 1. In particular, the $P_{V}$ profiles of all Balmer lines from $\mathrm{H} \epsilon$ down to $\mathrm{H} 10$ show a negative peak of $\sim-0.40 \%$, and the $P_{V}$ profile of the Ca II K line shows a negative peak of $\sim-0.7 \%$. Our $\mathrm{H} \delta$ line shows a milder polarimetric feature than that found by Hubrig et al. (2012), with a negative peak of $\sim-0.3 \%$ instead of $\sim-0.4 \%$. Our corresponding $\left\langle B_{z}\right\rangle$ value obtained from $\mathrm{H}$ Balmer lines is $-325 \pm 105 \mathrm{G}$, to be compared with their $-400 \pm 50 \mathrm{G}$.

Note that the bottom-left panel of our Fig. 6 shows that the linear fit exhibits a very uneven/skewed point distribution. This fully reflects the fact that the observed Stokes $V$ signal is not due to Zeeman effect (which would be responsible for a $P_{V}$ signal symmetric about the interpolating line). We note that our smallest error bar is larger than that found by Hubrig et al. (2012); this discrepancy is probably due to the fact that we have rescaled our error bar with the square-root of the reduced $\chi^{2}$, as explained in Bagnulo et al. (2012); indeed, by simply using the internal error bar, i.e., without re-scaling with the $\chi^{2}$, our error bar would be the same as that published by Hubrig et al. (2012), and in both cases of Figs. 6 and 8 we would obtain a $6 \sigma$ detection.
The null profile of our Fig. 6 is totally different from that published by Hubrig et al. (2012): our $N_{V}$ profile shows several spikes not visible in Hubrig et al. (2012), and, similarly to the case of Fig. 5, leads to a $4.7 \sigma$ field detection $\left(\left\langle N_{z}\right\rangle=-355 \pm\right.$ $75 \mathrm{G})$. The reasons of this puzzle are investigated in the next section.

\subsection{The non-uniqueness of the null profiles}

Null profiles have been introduced by Donati et al. (1997), and their definition and meaning were discussed by Bagnulo et al. (2009). In the context of the double difference method, the null profiles can be regarded simply as the difference between the $P_{V}$ profiles obtained from the combination of different pairs of frames. Let us assume that $i$ labels the frames obtained with the retarder waveplate at $-45^{\circ}$, and $j$ labels the frames obtained with the retarder waveplate at $+45^{\circ}$, and that these indices run in a temporal sequence (i.e., $i=2$ is taken after $i=1$ and so on). In case of HD 92207, we have $i=1, \ldots, 4$ and $j=1, \ldots, 4$, hence we can calculate sixteen different $P_{V}^{i j}$ profiles (although only four totally independent of each other); more in general, from $N$ pairs of frames one can obtain $N^{2}$ individual $P_{V}^{i j}$ profiles. While their average $P_{V}$ profile is unique, the definition of the null profile, if it is not explicitly linked to a temporal sequence, remains ambiguous, as one may calculate

$(1 / 8)\left[\left(P_{V}^{11}-P_{V}^{22}\right)+\left(P_{V}^{33}-P_{V}^{44}\right)\right]$, or

$(1 / 8)\left[\left(P_{V}^{11}-P_{V}^{33}\right)+\left(P_{V}^{22}-P_{V}^{44}\right)\right]$, or

$(1 / 8)\left[\left(P_{V}^{12}-P_{V}^{23}\right)+\left(P_{V}^{34}-P_{V}^{41}\right)\right]$, etc.

From four pairs of frames, formally we can construct 36 different $N_{V}$ profiles, and from $N$ pairs of frames, the number of different null profiles $N_{V}^{i j}$ that can be calculated by reshuffling the order of the frames is

$\left(\frac{N !}{[(N / 2) !]^{2}}\right)^{2}$

(or half of this number if we consider as identical the null profiles that have the same absolute value). For simplicity, one can consider only the cases where $i=j$. This way we can still construct $N P_{V}^{j j}$ profiles, and $N ! /[(N / 2) !]^{2} N_{V}^{j j}$ profiles (or half this number if we consider as identical those null profiles with identical absolute value). Figure 7 shows the four $P_{V}^{j j}$ profiles that can be obtained from the observations of HD 92207. Note that there are strong similarities between the $P_{V}^{11}$ and $P_{V}^{33}$ profiles, as well as between the $P_{V}^{22}$ and $P_{V}^{44}$ profiles. Therefore the null profile calculated following the temporal distribution of the frames, i.e. $(1 / 8)\left[\left(P_{V}^{11}-P_{V}^{22}\right)+\left(P_{V}^{33}-P_{V}^{44}\right)\right]$, shows a spike. On the other hand, a null profile calculated for example as

$(1 / 8)\left[\left(P_{V}^{11}-P_{V}^{33}\right)+\left(P_{V}^{22}-P_{V}^{44}\right)\right]$, or

$(1 / 8)\left[\left(P_{V}^{11}-P_{V}^{33}\right)+\left(P_{V}^{44}-P_{V}^{22}\right)\right]$

would be much smoother, because $P_{V}^{11} \approx P_{V}^{33}$ and $P_{V}^{22} \approx P_{V}^{44}$. To produce Fig. 6 we have used what we consider the most natural approach, i.e., we have kept the temporal order of the files, and calculated

$$
\begin{aligned}
N_{V} & =\frac{1}{8} \sum_{j=1}^{4}(-1)^{j}\left[\left(\frac{f^{\|}-f^{\perp}}{f^{\|}+f^{\perp}}\right)_{-45_{j}^{\circ}}-\left(\frac{f^{\|}-f^{\perp}}{f^{\|}+f^{\perp}}\right)_{+45_{j}^{\circ}}\right] \\
& =\frac{1}{8} \sum_{j=1}^{4}(-1)^{j} P_{V}^{j j}
\end{aligned}
$$




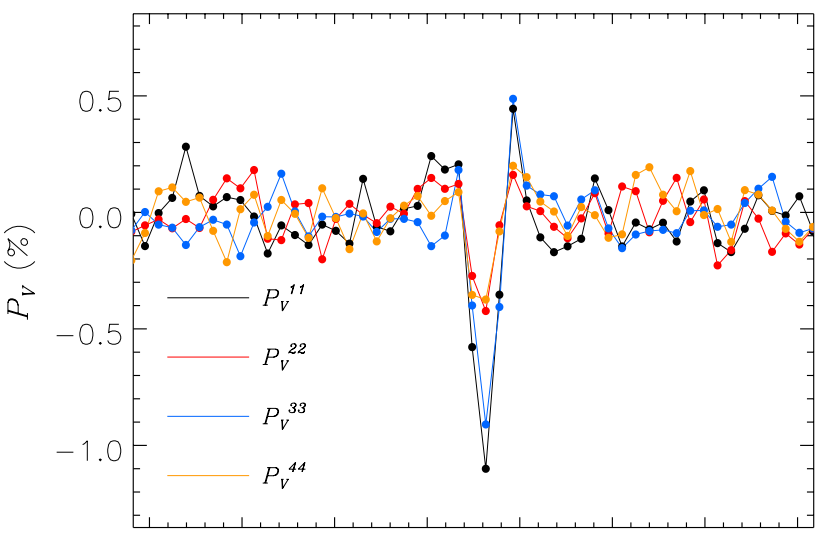

39153920392539303935394039453950 wavelength $(\AA)$

Fig. 7. Single rectified $P_{V}^{j j}$ profiles, in the region of the Ca II K line, obtained using a slightly imperfect wavelength calibration. The profiles are numbered as in Fig. 4. Note that this plot was obtained with a less accurate wavelength calibration than the plot shown in the rightmost panel of Fig. 4.
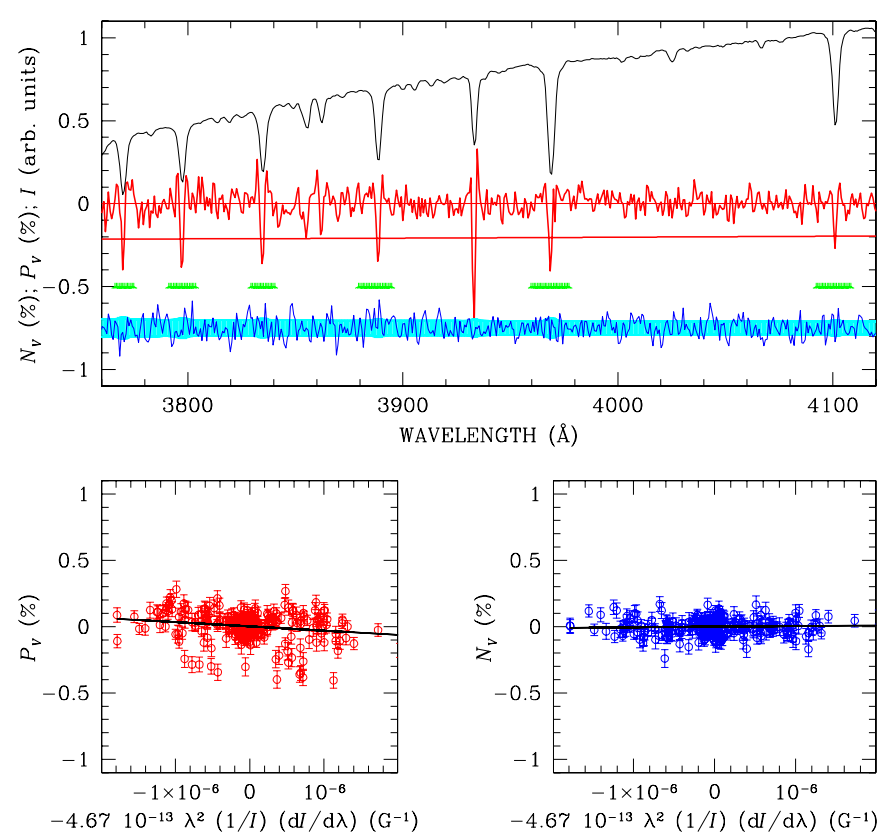

Fig. 8. As Fig. 6, but reshuffling the order of the files as explained in the text. Spikes are now visible only in the $P_{V}$ profile, while the $N_{V}$ profile appears smoother than in Fig. 6, and the corresponding $\left\langle N_{z}\right\rangle$ value is now $45 \pm 65 \mathrm{G}$.

where for $\alpha=-45^{\circ}, j=1,2,3,4$ correspond to the frames obtained at UT 03:56:53, 04:01:08, 04:02:12, and 04:06:29, respectively, and for $\alpha=+45^{\circ}, j=1,2,3,4$ correspond to the frames obtained at UT 03:58:28, 03:59:32, 04:03:49, and 04:04:54, respectively. By reshuffling the order of the frames as explained above, we could obtain a much smoother $N_{V}$ profile, qualitatively more similar to the one presented by Hubrig et al. (2012). Figure 8 shows the null profile obtained from Eq. (3) by imposing that for $\alpha=-45^{\circ}, j=1,2,3,4$ correspond to the frames obtained at UT 03:56:53, 04:02:12, 04:06:29, and 04:01:08, respectively, and that for $\alpha=+45^{\circ}, j=1,2,3,4$ correspond to the frames obtained at UT 03:58:28, 04:03:49, 04:04:54, and 03:59:32, respectively. The corresponding null field value is $\left\langle N_{z}\right\rangle=45 \pm 65 \mathrm{G}$. In the case of our best data-reduction of Fig. 5, from the null profile calculated after reshuffling the frames as explained above, the field detection in the null profile becomes $\left\langle N_{z}\right\rangle=90 \pm 65 \mathrm{G}$.

A similar situation would be encountered in the context of the double-ratio method (with the further ambiguity that two different definitions of the null profile can be given, see Bagnulo et al. 2009). Therefore we conclude that in case of multiple exposures, inspection to the scattering between the $P_{V}^{j j}$ profiles obtained from different pairs of frames (or even better the standard error of the mean normalised by the photon-noise error bar) may be a quality check more effective than, or at least complementary to the analysis of the null profiles. For an alternative and more sophisticated approach involving a $\sigma$-clipping algorithm of the $P_{V}^{i j}$ profiles we refer to Bagnulo et al. (2006).

\subsection{High resolution spectropolarimetry of HD 92207}

Four high-resolution spectropolarimetric observations of HD 92207 were obtained in February 2013 with the HARPSpol instrument (Piskunov et al. 2011) at the ESO 3.6-m telescope. These circular polarisation spectra, collected in the context of the ESO programme 090.D-0256(A), cover the 3800-6910 A wavelength domain at the resolution of $\lambda / \Delta \lambda=109000$. The typical signal-to-noise ratio $(\mathrm{S} / \mathrm{N})$ of these data is $300-400$ per pixel, measured at $\lambda \approx 5200 \AA$.

Each Stokes $V$ observation consisted of a series of 4 subexposures, obtained with the quarter-wave retarder plate angles of $45^{\circ}, 135^{\circ}, 225^{\circ}$, and $315^{\circ}$ relative to the beamsplitter. The spectra were extracted using the dedicated HARPSpol pipeline based on the REDUCE code of Piskunov \& Valenti (2002). Individual beams were normalised to the continuum and then combined to produce the Stokes $V$ and diagnostic null spectra using the ratio method outlined by Bagnulo et al. (2009). Further details about the reduction of HARPSpol data can be found in Makaganiuk et al. (2011).

No evidence of polarisation signatures was found in any individual spectral lines, therefore we applied the least-squares deconvolution method (Donati et al. 1997) to obtain high precision mean Stokes $I, V$, and null profiles. Using atmospheric parameters derived for HD 92207 by Przybilla et al. (2006), we extracted 355 metal lines deeper than $10 \%$ of the continuum from the VALD database (Kupka et al. 1999) and calculated LSD profiles with the code by Kochukhov et al. (2010). The resulting mean intensity and polarisation profiles are presented in Fig. 9. Despite an increase of $\mathrm{S} / \mathrm{N}$ by a factor of $\sim 20$, no Stokes $V$ signatures are detected. The null LSD profiles are also featureless. On the other hand, significant night to night variation of the mean Stokes $I$ profiles can be clearly seen.

Table 1 summarises individual HARPSpol spectropolarimetric observations of HD 92207. The four $\left\langle B_{z}\right\rangle$ estimates that we have obtained show no evidence of the magnetic field, consistent with the lack of signatures in the LSD Stokes $V$ profiles. The typical error bar of the mean longitudinal magnetic field derived from the high-resolution spectra is $10 \mathrm{G}$.

Returning to the individual spectral lines, we note that the intensity profile of the Ca II K line for which Hubrig et al. (2012) reported a circular polarisation amplitude of up to $1 \%$, exhibits a peculiar shape in our high-resolution spectra. As illustrated by Fig. 10, the observed $\mathrm{Ca}$ line is noticeably broader and deeper than predicted by the synthetic spectrum matching the stellar parameters determined by Przybilla et al. (2006). Furthermore, the line core is asymmetric and shows a double structure. The 

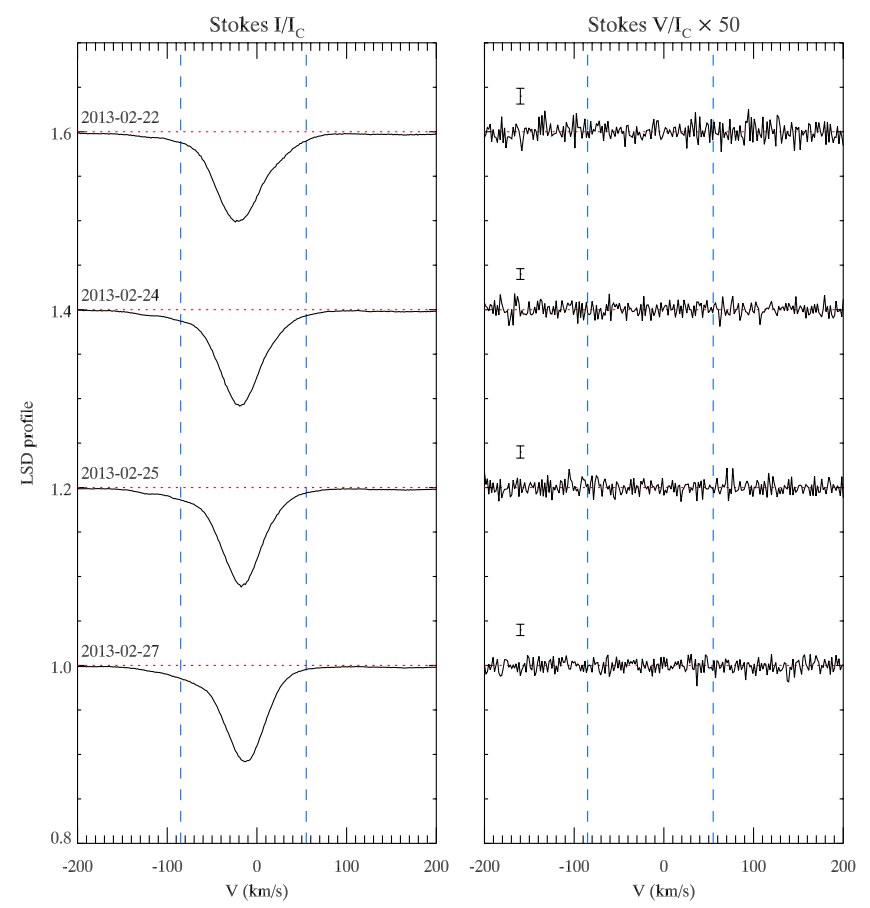

Fig. 9. Stokes $I$ and $V$ LSD profiles derived from the HARPSpol spectra of HD 92207. Observations obtained on different nights are offset vertically. The Stokes $V$ profiles are expanded by a factor of 50 relative to Stokes $I$. The vertical dashed lines indicated the velocity range used for $\left\langle B_{z}\right\rangle$ calculation.

Table 1. HARPSpol observations of HD 92207.

\begin{tabular}{lccccr}
\hline \hline HJD & $S / N$ & $S / N$ & $\left\langle B_{z}\right\rangle$ & $\left\langle N_{z}\right\rangle$ & $F A P$ \\
-2450000 & $(/ \mathrm{px})$ & $(\mathrm{LSD})$ & $(\mathrm{G})$ & $(\mathrm{G})$ & $(V)$ \\
\hline 6345.6732 & 300 & 5629 & $0.2 \pm 13.4$ & $2.0 \pm 13.4$ & 0.897 \\
6347.6720 & 440 & 8222 & $-19.1 \pm 9.5$ & $18.9 \pm 9.5$ & 0.020 \\
6348.6735 & 390 & 7299 & $11.8 \pm 10.7$ & $-21.4 \pm 10.7$ & 0.402 \\
6350.6660 & 420 & 7893 & $-1.8 \pm 10.1$ & $-13.8 \pm 10.1$ & 0.147 \\
\hline
\end{tabular}

Notes. Columns give the heliocentric Julian date of observations, the peak $\mathrm{S} / \mathrm{N}$ per pixel of Stokes $V$ spectra, the $\mathrm{S} / \mathrm{N}$ achieved in the LSD profiles, and the estimates of the mean longitudinal magnetic field derived from Stokes $V$ and diagnostic null LSD profiles. The final column gives the false alarm probability that the structure in the mean circular polarisation profile can be attributed to random noise. Marginal detection corresponds to $F A P<10^{-3}$.

Ca II H line exhibits the same profile. Similarly, the resonance $\mathrm{Na}$ I D lines are also very deep and show a two-component structure. It appears that all these strong features are formed in the circumstellar environment or in corotating wind structures (Ignace et al. 2009) rather than in the stellar atmosphere. No circular polarisation is detected in any of our HARPS spectra, consistently with our suggestion that the signal measured with FORS is spurious.

Finally, the black and red lines of Fig. 11 show the profiles observed in the parallel beam with retarder waveplate at $45^{\circ}$ and $225^{\circ}$, respectively. These profiles appear constant within photon-noise error bars. The blue line (blue-shifted by $24 \mathrm{px}$ with respect to HARPS observations) shows how the offset observed with FORS between profiles 5 and 8 in Fig. 3 would appear if observed at the spectral resolution of HARPS. In conclusion, HARPS data suggest that the offsets observed with FORS are not due to stellar variability.

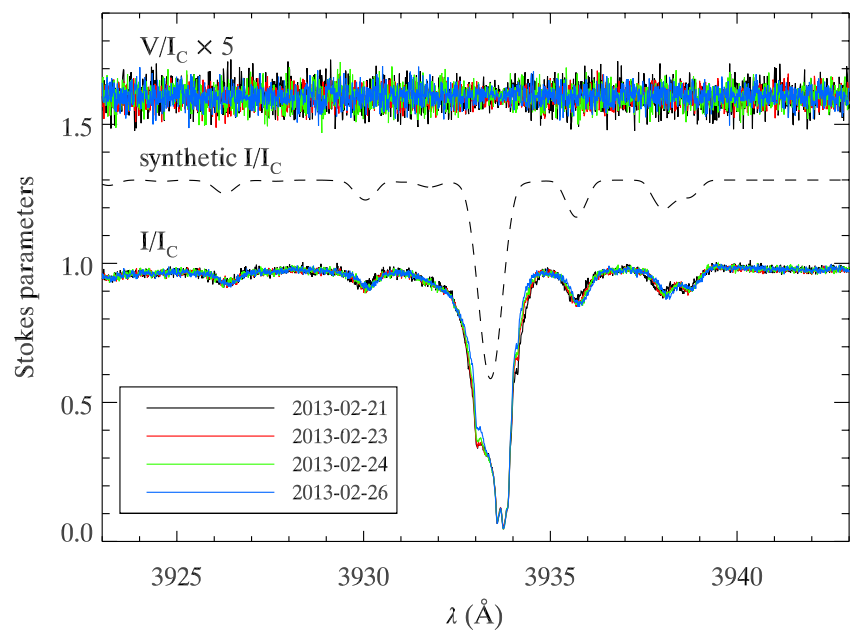

Fig. 10. High-resolution Stokes $I$ and $V$ spectra of HD 92207 in the vicinity of the Ca II $\mathrm{K}$ line. Individual observations (bottom solid lines) are compared to the synthetic spectrum (mid dashed line). The latter is offset vertically by +0.3 for display purpose. Stokes $V / I_{\mathrm{c}}$ profiles (upper solid lines) are multiplied by a factor of 5 and offset by +1.6 .

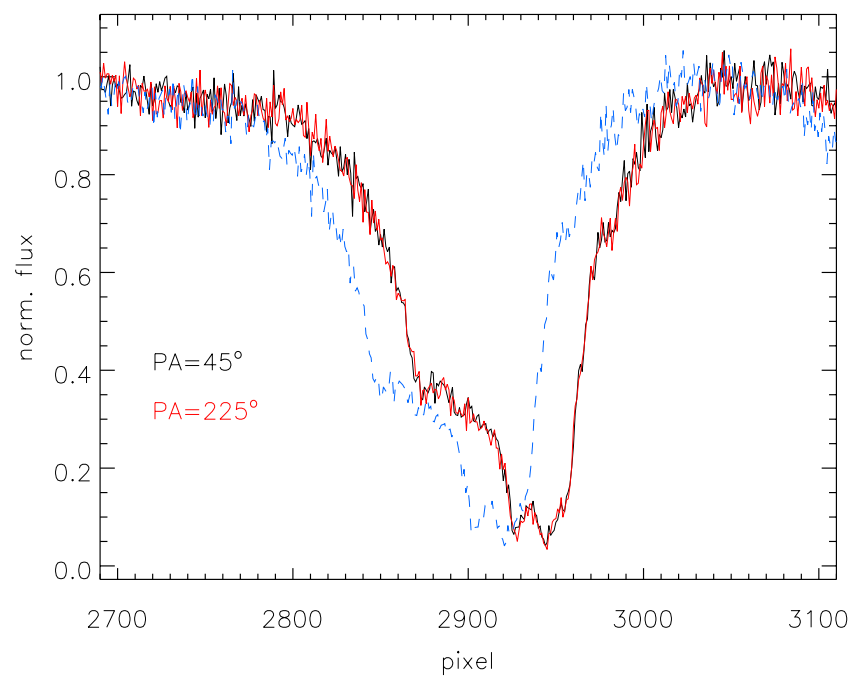

Fig. 11. Profiles of the Ca II K line extracted from the HARPS frames of the HJD 2456347 (parallel beam). The blue line shows how the offset observed with FORS would appear in HARPS data if it were due to variability intrinsic to the star.

\section{Discussion and conclusions}

There has been a debate in the literature regarding the reliability of some magnetic field measurements obtained with the FORS instrument of the ESO VLT during various surveys. In particular, there have been numerous cases of field detection obtained with FORS that were not confirmed by follow-up with the same or other instruments. This situation has been discussed in detail by Bagnulo et al. (2012), who have concluded that 1 ) in some cases FORS data have not been fully correctly reduced; and 2) photon-noise is not the only source of measurement error. In particular, Bagnulo et al. (2012) have proposed that very small instrument instabilities, probably unavoidable in slit-fed Cassegrain-mounted instruments, may contribute a nonnegligible fraction of the actual field measurement error bar. In this paper we have addressed this issue in greater detail, using simple numerical simulations as well as calibration and science data. 
Our numerical simulations show for instance that offsets as small as $1 \%$ of the FWHM may lead to a substantial spurious polarisation signal in deep spectral lines. The beam swapping technique may cancel out such spurious effects if the offsets are both stable and systematic (i.e., always affecting the parallel or the perpendicular beam, or both beams obtained at a certain position angle of the retarder waveplate, in the same way). However, certain combinations of systematic offsets, or simply random offsets, may lead to spurious polarisation signals even when observations are obtained with beam swapping.

The presence of polarimetric optics (with moving parts) introduces offsets. In FORS, the Wollaston prism is responsible for a 0.55 px offset between the parallel and the perpendicular beam; when the retarder waveplate rotates from $-45^{\circ}$ to $+45^{\circ}$, both beams are blue-shifted by about 0.13 px (in our case $\sim 0.1 \AA$ ). These combined effects cannot be compensated by the beam swapping technique, and must be corrected by a careful wavelength calibration. Practically, our experience shows that while it is mandatory to calibrate independently the parallel and the perpendicular beam, it is safer to use the same pair of wavelength calibrations for all frames obtained at different position angles of the retarder waveplate. The two wavelength solutions must be obtained adopting exactly the same fitting function and using exactly the same arc lines. We have also noted that different offsets are introduced when the retarder waveplate is rotated at $135^{\circ}$ and $225^{\circ}$, which suggests that more accurate results may be achieved by observing with the retarder waveplate set at two position angles only, instead of four.

The positions of the spectra are not perfectly reproducible when the retarder waveplate is removed and re-inserted: global offsets of $\sim 0.1 \mathrm{px}$ have been observed when comparing calibration data obtained in different days. This suggests that, to achieve the highest accuracy, scientific frames can be safely combined only if the observing series has not been interrupted to remove and re-insert the retarder waveplate (or any other optical component, such as the grism). If the instrument setting is changed before the series is completed (other than by the rotation of the retarder waveplate), we recommend that the entire series be repeated. We are not able to assess whether random offsets may occur following a rotation of the retarder waveplate, but we found no evidence of this. We note that a potential global offset between daytime calibrations and night time scientific frames does not represent a problem because the offset between parallel and perpendicular beam remains constant, and may be corrected.

We have found strong evidence for small and presumably random offsets in the FORS observations of HD 92207, a supergiant A0 star in which Hubrig et al. (2012) have recently claimed a field detection, and that we have adopted here as a case study. Spectra from the same beam, obtained with the retarder waveplate in identical positions, appear shifted by up to $\sim 0.25 \mathrm{px}$ from each other. In the context of ultra-high signalto-noise ratio spectro-polarimetric data, these random offsets are not negligible and contribute significantly to the error budget. However, the actual magnitude of the observed offsets is very small $\left(\sim 0.03^{\prime \prime}\right)$, hence it is not easy to pin down their origin. While they might be linked to the non-reproducibility of the position of the spectral lines as the retarder waveplate moves, they might well have a completely different origin. In general, they could be due to the star itself (rapid pulsation or rapid changes of the radial velocity), but this (unlikely) hypothesis seems ruled out completely by the fact that similar offsets are not present in our HARPS data. They could be due to differential instrument flexures (see a detailed discussion in Bagnulo et al. 2012), and/or to movements of the stellar image in the slit due either to seeing or to guiding inaccuracy. Actually, since FORS observations of HD 92207 were obtained under excellent seeing conditions $\left(\sim 0.7^{\prime \prime}\right)$ and with extremely short integration time $(t=3 \mathrm{~s}$ each exposure), image movements due to atmospheric variability are likely. Another potential cause is a time dependent differential atmospheric diffraction. During the observations, the slit was nearly perpendicular to the parallactic angle, and between the beginning and the end of the exposures, the airmass varied from 1.55 to 1.65 . Airmass 1.55 is the limit up to which the Longitudinal Atmospheric Dispersion Corrector (Avila et al. 1997) may fully compensate. However, we could not confirm that the observed offset is wavelength dependent, as is atmospheric dispersion. Clearly, the observed offsets may be due to a combination of all the effects mentioned above. More insight could be provided with the help of a systematic investigation into the archive data, and few technical tests (e.g., measuring the stability of the centroid of the star imaging obtained through the slit, with no grism inserted; obtaining a series of wavelength calibrations with the telescope tilted, while the instrument is rotating).

How do these instabilities affect the reliability of FORS measurements, and how can their impact be evaluated?

Null profiles may or may not reveal instrumental problems, and we should recall that they have mainly a statistical meaning. Using the longitudinal field values $\left\langle N_{z}\right\rangle$ calculated from the null profiles for a very large sample of FORS1 data, Bagnulo et al. (2012) concluded that, statistically, the actual field error bars are up to $50 \%$ larger than those calculated from photon-noise only. However, when looking at an individual measurement, an $\left\langle N_{z}\right\rangle$ value consistent with zero does not automatically imply that the corresponding $\left\langle B_{z}\right\rangle$ value is not altered by instrumental (or datareduction) effects. Figure 8 is a convincing example of how a combination of instrument instabilities and non-perfect wavelength calibration leads to a number of circular polarisation features associated with a clean null profile.

In this paper we have also shown that if the observing series comprises more than two pairs of frames, the definition of null profile does not have a unique interpretation, and null profiles obtained by sorting the frames in different ways are different. Therefore, especially in case of dubious detections, we recommend calculating and inspecting the scatter of the $P_{V}$ profiles obtained from different pairs of frames (e.g., one could plot the ratio between the standard error of the mean and the photonnoise error bar). Another important test is to compare the spectra recorded in the same beam and obtained with the retarder waveplate at identical position, and to check if they are consistent within photon-noise error bars.

In spite of all the issues discussed in this paper, we should remark that if data are treated correctly, spurious detections are relatively unlikely to occur. In our case study, we could reproduce a previously reported field detection only by degrading the accuracy of our wavelength calibration. Even in that case, applying a $\sigma$ clipping algorithm to the least-square fit would bring our field value to less than $3 \sigma$ from zero provided the external error is adopted as uncertainty estimate. Finally, we recall that the spikes of the $P_{V}$ profile shown in Fig. 8 should not be mistaken for Zeeman signatures. A genuine Zeeman signature should exhibit a typical S profile (except when longitudinal field is reversing sign and the star rotates rapidly, but this cross-over effect can be revealed only at higher spectral resolution), and the zeroorder moment of Stokes $V$ about line centre should always be zero.

We should also note that in this paper we have made no attempts to establish how frequently instabilities occur at a significant level. Accordingly, there is no implication that the offsets 
at the level detected in the observations of HD 92207 are typical of FORS1/2 measurements.

In fact, the considerations presented in this paper are not restricted to the FORS instrument, but are of a much more general relevance. It is not unusual that instruments are used for tasks for which they were not originally designed, and in these cases it is up to the user to demonstrate that instrumental sensitivity corresponds to its accuracy. Calculating the square-root of the photon count is a very simple and fundamental task, but not necessarily sufficient to evaluate the reliability of astronomical measurements.

Acknowledgements. We thank Naum Rusomarov for performing the HARPSpol observations of HD 92207. J.D.L. acknowledges financial support for this project from the Natural Sciences and Engineering Research Council of Canada. O.K. is a Royal Swedish Academy of Sciences Research Fellow, supported by grants from Knut and Alice Wallenberg Foundation and Swedish Research Council. We thank the anonymous referee and Gregg Wade for very useful comments to the manuscript.

\section{References}

Alecian, E., Wade, G. A., Catala, C., et al. 2013, MNRAS, 429, 1001 Appenzeller, I., Fricke, K., Furtig, W., et al. 1998, The Messenger, 94, 1 Aurière, M., Wade, G. A., Konstantinova-Antova, R., et al. 2009, A\&A, 504, 231

Avila, G., Rupprecht, G., \& Beckers, J. M. 1997, Proc. SPIE, 2871, 1135

Bagnulo, S., Szeifert, T., Wade, G. A., Landstreet, J. D., \& Mathys, G. 2002, A\&A, 389, 191
Bagnulo, S., Landstreet, J. D., Mason, E., et al. 2006, A\&A, 450, 777 Bagnulo, S., Landolfi, M., Landstreet, J. D., et al. 2009, PASP, 121, 993

Bagnulo, S., Landstreet, J. D., Fossati, L., \& Kochukhov, O. 2012, A\&A, 538, A129

Catala, C., Alecian, E., Donati, J.-F., et al. 2007, A\&A, 462, 293

Donati, J.-F., \& Landstreet, J. D. 2009, ARA\&A, 47, 333

Donati, J.-F, Semel, M., Carter, B. D., Rees, D. E., \& Collier Cameron, A. 1997, MNRAS, 291, 658

Grunhut, J. H., \& Wade, G. A. 2012, ASPC, 465, 42

Grunhut, J. H., Wade, G. A., Hanes, D. A., \& Alecian, E. 2010, MNRAS, 408, 2290

Henrichs, H., de Jong, J. A., Donati, J.-F., et al. 2000, in The Be Phenomenon in Early-Type Stars, eds. M. A. Smith, H. F. Henrichs, \& J. Fabregat, ASP Conf. Ser., 214, 324

Hubrig, S., Schoeller, M., Kholtygin, A. F., et al. 2012, A\&A, 546, L6

Ignace, R., Hubrig, S., \& Schöller, M. 2009, AJ, 137, 3339

Izzo, C., de Bilbao, L., Larsen, J., et al. 2010, Proc. SPIE, 7737, 773729

Jordan, S., Bagnulo, S., Werner, K., \& O'Toole, S. J. O. 2012, A\&A, 542, A64

Kochukhov, O., Makaganiuk, V., \& Piskunov, N. 2010, A\&A, 524, A5

Kupka, F., Piskunov, N., Ryabchikova, T. A., Stempels, H. C., \& Weiss, W. W. 1999, A\&AS, 138, 119

Landstreet, J. D., Bagnulo, S., Fossati, L., Jordan, S., \& O’Toole, S. J. O. 2012, A\&A, 541, A100

Makaganiuk, V., Kochukhov, O., Piskunov, N., et al. 2011, A\&A, 525, A97

Neiner, C., Hubert, A.-M., Frémat, Y., et al. 2003, A\&A, 409, 275

Piskunov, N. E., \& Valenti, J. A. 2002, A\&A, 385, 1095

Piskunov, N., Snik, F., Dolgopolov, A., et al. 2011, The Messenger, 143, 7

Przybilla, N., Butler, K., Becker, S. R., \& Kudritzki, R. P. 2006, A\&A, 445, 1099

Silvester, J., Neiner, C., Henrichs, H. F., et al. 2009 MNRAS, 398, 1505

Sterzik, M. F., Bagnulo, S., \& Pallé, E. 2012, Nature, 483, 64

Wade, G. A., Drouin, D., Bagnulo, S., et al. 2005, A\&A, 442, L31

Wade, G. A., Bagnulo, S., Drouin, D., Landstreet, J. D., \& Monin, D. 2007, MNRAS, 376, 1145 